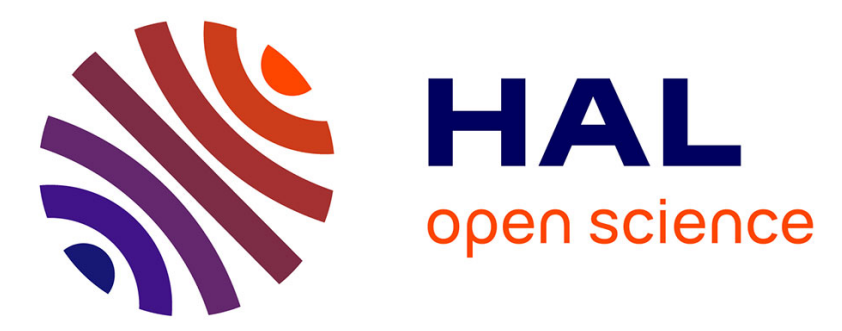

\title{
Compensation of position offset of acoustic transducers using compressive sensing concept
}

Irina Murgan, Angela Digulescu, Ion Candel, Cornel Ioana

\section{To cite this version:}

Irina Murgan, Angela Digulescu, Ion Candel, Cornel Ioana. Compensation of position offset of acoustic transducers using compressive sensing concept. OCEANS 2016 - OCEANS '16 MTS/IEEE. The Sea's the Limit, Sep 2016, Monterey, United States. pp.1-4, 10.1109/OCEANS.2016.7761083 . hal01441747

\section{HAL Id: hal-01441747 \\ https://hal.science/hal-01441747}

Submitted on 20 Jan 2017

HAL is a multi-disciplinary open access archive for the deposit and dissemination of scientific research documents, whether they are published or not. The documents may come from teaching and research institutions in France or abroad, or from public or private research centers.
L'archive ouverte pluridisciplinaire HAL, est destinée au dépôt et à la diffusion de documents scientifiques de niveau recherche, publiés ou non, émanant des établissements d'enseignement et de recherche français ou étrangers, des laboratoires publics ou privés. 


\title{
Compensation of position offset of acoustic transducers using compressive sensing concept
}

\author{
Irina Murgan ${ }^{1}$, Angela Digulescu ${ }^{1,2}$, Ion Candel $^{1}$, Cornel Ioana ${ }^{1}$ \\ ${ }^{1}$ University of Grenoble-Alpes, GIPSA-lab, Saint Martin D'Hères, France \\ ${ }^{2}$ Military Technical Academy, Bucharest, Romania \\ [irina.murgan, angela.digulescu, ion.candel, cornel.ioana]@gipsa-lab.grenoble-inp.fr
}

\begin{abstract}
This paper presents a new technique for acoustic transducers position offset compensation, based on compressive sensing reconstruction in the warped domain. In underwater acoustics, the transducers relative position is important for applications involving direction of arrival estimation, localization or source detection. When the transmitter-receiver transducers configuration is inappropriate, the received signal's informational content is not the same as the one of the emitted signal. In the case of applications such as underwater objects tracking, the experimental setup constraints and the water flow operational conditions lead to the perturbation of the emitted and received signals propagation. We use compressive sensing reconstruction of the received signal, in the warped domain, in order to recover its missing spectral information due to wave's propagation. Tests were conducted in a reduced scale experimental facility, in order to prove the interest of using the signal compressive sensing recovery for the signal's time of arrival estimation and to quantify the improvement introduced by this signal processing method. The results show that the time of arrival estimation can be considerably improved after the received signal's samples recovery, with the matched filter response improvement.
\end{abstract}

\section{Keywords - compressive sensing; warping transform; time of arrival estimation; matched filter}

\section{INTRODUCTION}

In underwater acoustics, waves propagation and reception are conditioned by factors related to wave's propagation physics (such as attenuation, absorption, scattering, geometrical spreading or propagation losses) and to geometrical set-up of the acoustic system. In this second category, the acoustic transducers inappropriate alignment is one of the frequently encountered situation. In most underwater applications, due to underwater unsteady conditions, vibrations, or geometrical misalignments, a position offset of the acoustic transducers appears. In terms of receiving signals, the misalignments between sensors conduct to the loss of the signal's energy and can affect the processing at the receiver level. The purpose of this study is to propose a method to compensate the signal's amplitude loss due to this offset.

The acoustic transducers position offset impacts the transmitted information between the emitter and the receiver: the wave's shape and content are corrupted (Figure 1). In this example, we consider the case of a transmitter and receiving sensors configuration, aimed to detect a migrating object in a zone of interest. The misalignment of the sensors conducts to the attenuation of the received signal affecting also the detection of the object.

Figure 2 illustrates examples of emitted and received signals in such case. In the ideal case when the emitting and the receiving sensors are perfectly aligned (Figure 2.a.), the received signal is just delayed and attenuated. Where the alignment is not well done (Figure 2.b.), the received signal has informational missing content. The informational content loss of the received signal impacts the signal processing results, such as the matched filter response, on which depends the signal's time of arrival (TOA) accuracy. Namely, the received signal will be not perfectly matched to the emitting one, affecting also the estimation of TOAs.

In consequence, it is important to maintain the signal's informational content between emission and reception, in order to preserve an optimal signal processing performing. Hence, the effects of transducers position offset need to be compensated to avoid dramatically errors of the data processing results. For some simple applications, with fixed experimental configurations, the position offset can be geometrically quantified and its compensation is trivial. In most cases, especially when multi-element configurations are used (as the example from figure 1), the position offset compensation demands more complex methods, defined around the adaptive beamforming techniques.

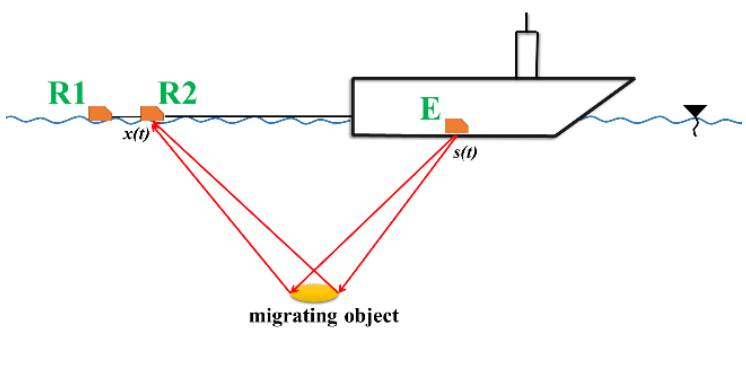

Figure 1. An example of the application principle representation.R1 is the misaligned transducer that is supposed to record the reflected wave, while the $\mathrm{R} 2$ is positioned where the wave is actually reflected 

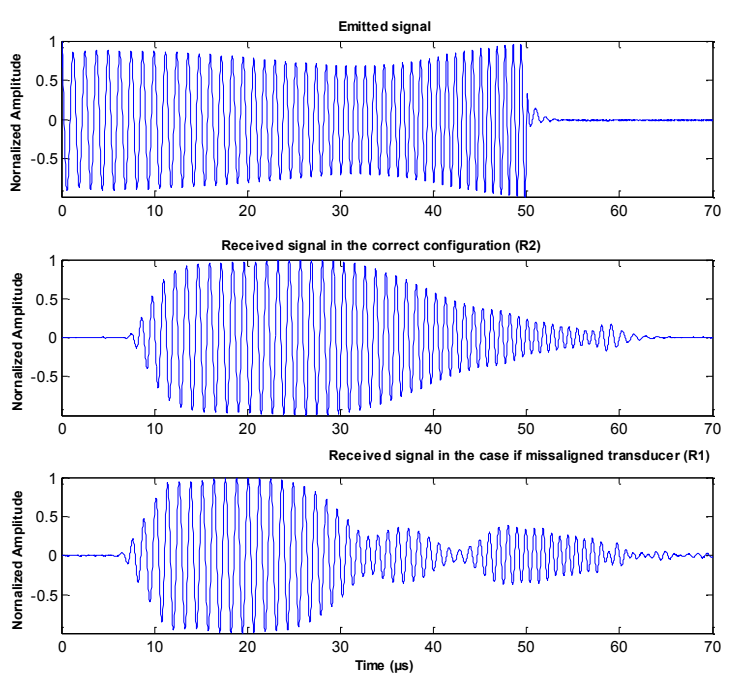

Figure 2. Emitted and received wave forms for the ideal (a) and real (b) cases.

This paper presents a method that contributes to improve the performances of a sensing array by recovering the lost samples, in the case of sensor misalignments.

In previous studies, the CS concept has been successfully used for separation of linear and nonlinear frequency modulation signals $[1,2]$. In this paper, the CS concept aiming is to reconstruct signals that have, in the frequency domain, a sparse basis representation. [3-5]. In a standard formulation of the CS concept, the observations are made in the time domain [6]. Here, we deal with signals that are sparse in the frequency domain and we apply the compressive sensing algorithm in a warped domain, in order to perform their reconstruction [2]. We consider that the emitted signals are linear frequency modulations (LFM), widely used in the sonar systems. As wide band signals, they can be characterized by instantaneous frequency laws (IFL). The IFL's coordinates are estimated using a phase tracking algorithm developed in [7], which will enable to express the warping transform function. A warping (or time axis) transform is used to represent the LFM signal into a stationary sinusoid in the warped domain (while in the frequency domain is sparse). The CS algorithm will be used to reconstruct the sinusoid missing information, then an unwarping transform will turn the warped signal basis into the original one.

The paper is organized as follows: the section 2 contains the description of the CS concept used for the received signal reconstruction, the section 3 presents the experimental test, the section 4 shows the obtained results. Finally, concluding remarks are exposed in section 5 .

\section{COMPRESIVE SENSING CONCEPT II.1. Problem formulation}

Consider a LFM transmitted signal defined by:

$$
s(t)=A e^{j\left(2 \pi f_{0} t+c t^{2}\right)}
$$

where $f_{0}$ is the central frequency and $c$ is the chirp rate. For reception, we consider the case of a signal corrupted and disturbed by the propagation in the environment and by the measurement configuration misalignments, which are the main sources of errors considered in our work.
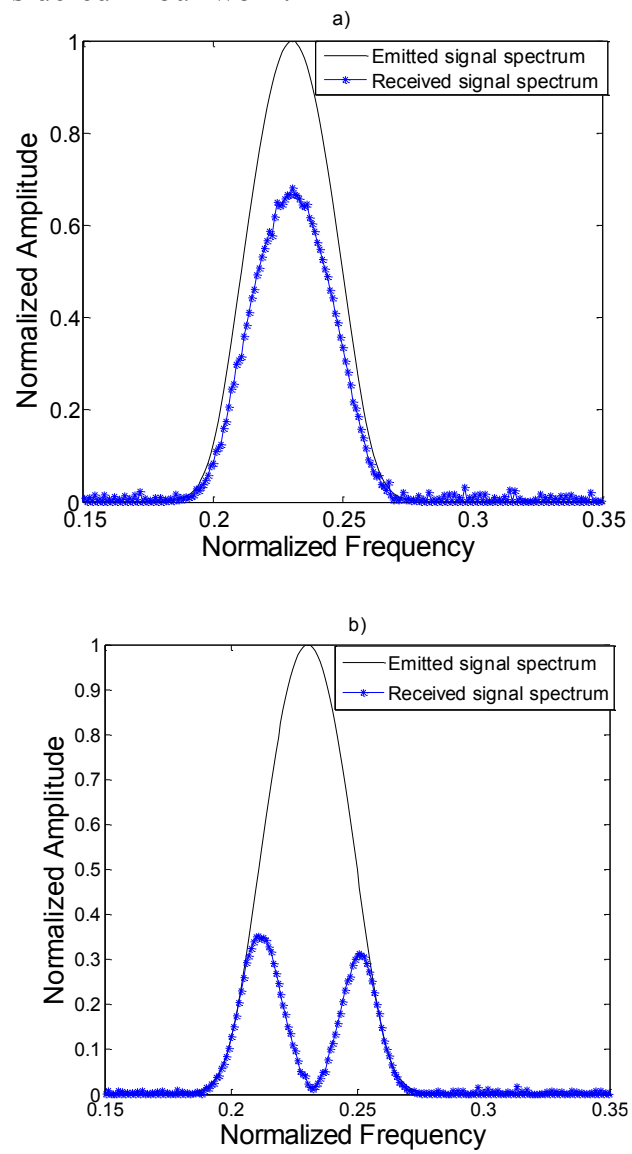

Figure 3. Example of emitted/received spectra in the case of perfectly aligned transducers (a) and emitted/received spectra, in the case of misaligned transducers (b).

The travelled environment by the acoustic wave (here the unsteady sea flow) will add noise to the received signal, $n(t)$, while the measurements configuration misalignments will impede a complete signal reception. Consider the received signal:

$$
x(t)=y(t)+n(t)
$$

where $y(t)$, is a fraction of $s(t)$, and it represents an incomplete received spectral information as illustrated by the Figure 3. Actually, the signal's propagation along different paths produces, at the receiver level, interferences that will conduct to the loss of some of the samples. The purpose of the warping based CS reconstruction is to recover part of theses samples, in order to ensure a good performances of the matching filtering stage.

\section{II.2. Warping based CS reconstruction}

As the transmitted signal is known, we will use the modulation law as an input parameter for the 
design of the appropriate representation space for samples recovering. In a general case, we consider an arbitrary IFL, $\varphi(t)$, and a finite set of coordinates $\left\{\varphi\left(t_{k}\right)\right\}_{k=\overline{1, L}}$, with $\mathrm{L}$ divided segments of the signal $x(t)$. We consider a discrete warping function $w_{d}(t)$ defined by the following equation system:

$$
\left\{\begin{array}{c}
\varphi\left(w_{d}\left(t_{k}\right)\right)=w_{d}\left(\varphi\left(t_{k}\right)\right)=t_{k}, k=\overline{1, L} \\
w_{k}^{(0)}=\frac{1}{2}\left(I_{k}+I_{k+1}\right)
\end{array}\right.
$$

where $w_{k} \stackrel{\text { def }}{=} w_{d}\left(t_{k}\right)$ and $\left[I_{k}, I_{k+1}\right]$ is centered around $t_{k}$. IFL has to be monotone on each of $L$ intervals. This fact will ensure the existence of the inverse warping function.

Thus, the local solution of this equation system, for a point $p$ is given by:

$w_{k}^{(p)}=w_{k}^{(p-1)}-\operatorname{sgn}\left(\varphi\left(w_{k}^{(p-1)}-t_{k}\right) \frac{I_{k}-I_{k-1}}{2^{p+1}}\right.$

with the solution convergence condition:

$$
\left|w_{k}^{(p+1)}-w_{k}^{(p)}\right|<\varepsilon
$$

We define a warping operator, $W$, as:

$$
\left\{W, w(t) \in C^{1}, w^{\prime}(t)>0: x(t) \rightarrow W(x(t))\right\}
$$

where $C^{1}$ is the class of differentiable functions. In order to be able to apply CS algorithm $[1,[2,[8]$ on the warped signal $W(x[n])$, the STFT of it is computed:

$$
\operatorname{STFT}[n, k]=\sum_{m=0}^{M-1} w[m] x[n+m] e^{-j 2 \pi m k / M}
$$

where $\mathrm{M}$ is the length of the of the Hamming window used to compute the STFT. To the obtained frequency vector, the CS algorithm in applied, and so the $W(x[n])$ reconstruction is achieved. In contrast with the processing steps performed that precede the CS reconstruction in $[1,2]$, here we do not perform LFM separations. Hence, the entire STFT vector is used to reconstruct the linearized frequency by the warping operator. After the CS reconstruction, the inverse warping operator is used to return in the original time domain.

\section{EXPERIMENTAL TESTS}

In order to demonstrate the results of the warped CS-based reconstruction algorithm, we have performed an experimental test in which we reproduce at reduce scale the phenomenon described in introduction (Figure 1). Thus, in a water tank, it is installed an emitter-receiver transducer system configuration and a target (migrating object). Figure 4.a illustrates the experimental set-up, while the figure 4.b shows the real bank test. A chirp with a frequency variation between $0.5 \mathrm{MHz}$ and $1.1 \mathrm{MHz}$ is emitted towards the migrating object ( $\mathrm{E}$ is the emitter) and the reflected signal is recorded with the receivers $\mathrm{R} 1$ and $\mathrm{R} 2$, with a sampling frequency of $50 \mathrm{MHz}$. Signals are displayed in figure 2.
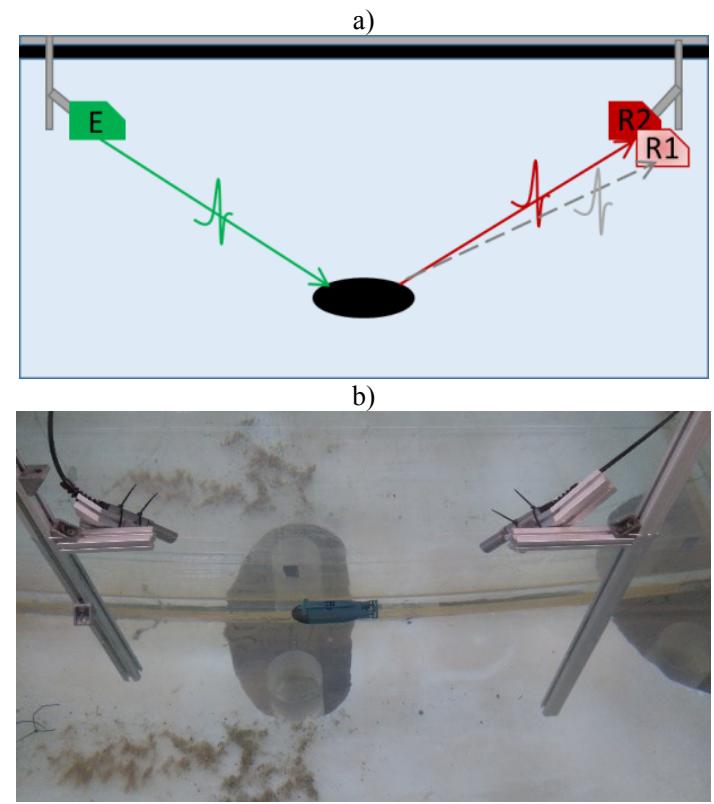

Figure 4. a) The experimental set-up and b) the real experimental configuration

The need to reconstruct the received signal is given by the fact that the TOA estimation is considerably affected by the transmitted information between the emission and reception. The TOA estimation is performed using matched filtering. Its bad response (as is the presented case in figure 5) will conduct to an imprecise TOA estimation.

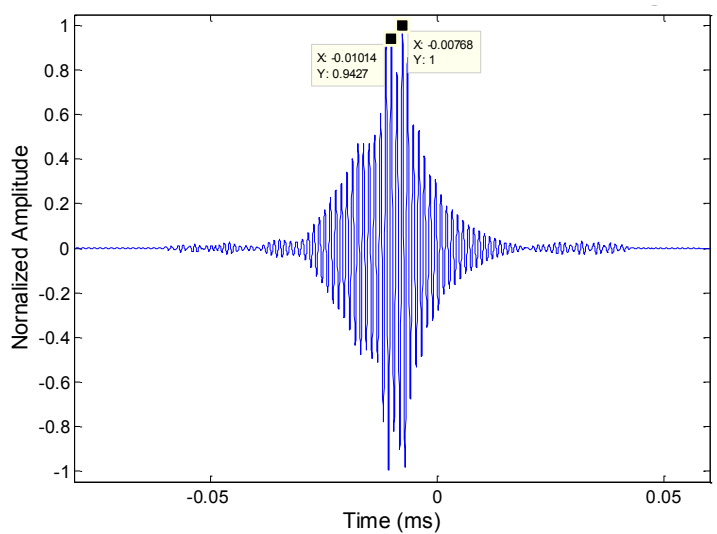

Figure 5. The result of the matched filter applied on the emitted signal and the received signal in the case of misaligned transducers

\section{RESULTS}

The purpose of the signal reconstruction consist in informational content recovery. If we compare the spectral content on the initial received signal with the recovered one, we can see an important improvement (Figure 6). 


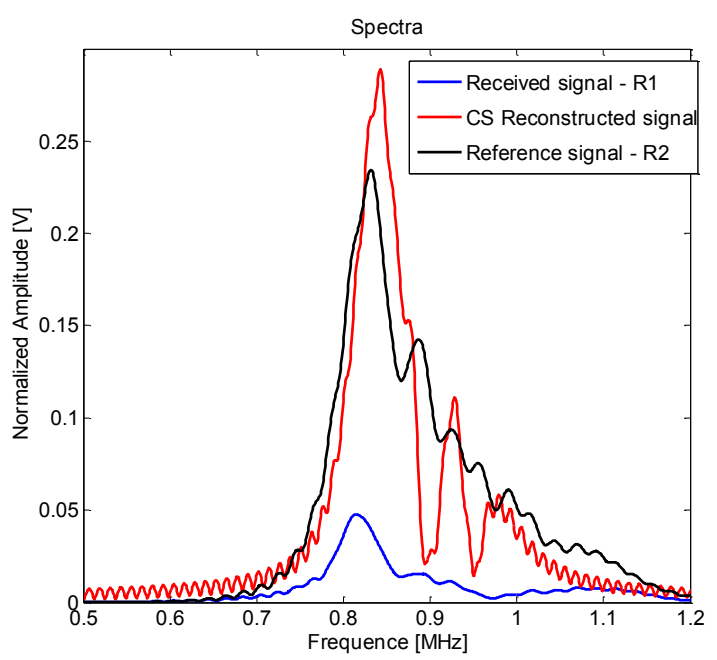

Figure 6. The spectrums of the: reference received signal in the proper configuration, E-R2, (in black line); received signal in the case of misaligned transducers, E-R1 (in blue line); the recovered signal (in red line)

This spectral recovery achieved by the warping based CS reconstruction algorithm, improves considerably the TOA based matched filter estimation since only one peak is detected, at the right position.

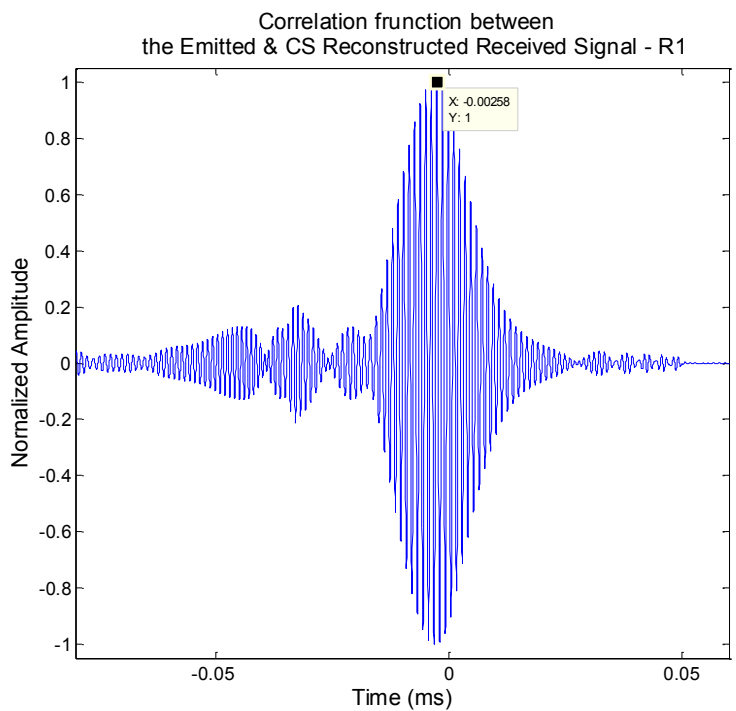

Figure 7. The matched filter response for the recovered signal

\section{CONCLUSION}

A warping based compressive sensing reconstruction method for wide band signals is presented. The general applicative context of this work is related to the cases where the waves propagating in dispersive environments are subject of interferences that produce the loss of some samples of the signals. A method to recover these samples is proposed in this paper, based on the use of compressive sensing reconstruction applied in the warped domain. Signal's recovery lead to better results in terms of matched filtering.

The perspectives are to extend the present work to different emitted frequency modulation waveforms. We look also to apply this study in the case of multi-transducer configurations in order to allow their application in other fields of research.

\section{ACKNOWLEDGEMENT}

This work is partially supported by the Montenegrin Ministry of Science, project grant "New ICT Compressive sensing based trends" applied to: multimedia, biomedicine and communications" (ACRONYM: CS-ICT).

\section{REFERENCES}

[1] I. Orovic, S. Stankovic, and L. Stankovic, Compressive sensing based separation of LFM signals, Proceedings ELMAR-2014. IEEE, pp. 1-4. 2014.

[2] L. Stankovic, I. Orovic, S. Stankovic, and M. Amin, Compressive Sensing Based Separation of Nonstationary and Stationary Signals Overlapping in Time-Frequency, IEEE Transactions on Signal Processing vol. 61, no. 18, pp. 45624572, Sep. 2013.

[3] S. Stankovic, I. Orovic, E. Sejdic, Compressive Sensing, Multimedia Signals and Systems, Springer 2012.

[4] R. Baraniuk, Compressive Sensing, IEEE SP Magazine vol. 24, no. 4, pp. 118-121, 2007.

[5] P. Flandrin, P. Borgnat, Time-Frequency Energy Distributions Meet Compressed Sensing, IEEE Trans. SP vol. 58, no. 6, pp. 2974-2982, 2010.

[6] B. Jokanovic, M. Amin, S. Stankovic, Instantaneous frequency and time-frequency signature estimation using compressive sensing, SPIE Defense, Security and Sensing, Baltimore, Maryland, United States, 2013.

[7] C. Ioana, C. Gervaise, Y. Stéphan, J.I. Mars, Analysis of underwater mammal vocalizations using time-frequency phase tracker, Applied Acoustics vol 71, no. 11, pp. 10701080, 2010.

[8] C. Bernard, C. Ioana, I. Orovic, S. Stankovic, Analysis of underwater signals with nonlinear time-frequency structures using warping-based compressive sensing algorithm, IEEE Oceans Conference, October 2015. 